\title{
REPORT ON À REFRESHER COURSE HELD BY THE NATAL COASTAL BRANCH OVER THE WEEKEND OF 11 TO 13 JULY 1980
}

In the first session Miss M. Ashby and Mrs. J. Morton, assisted by Miss A. Clayton, dealt with Maitland mobilisation of the cervical spine. A brief introduction covered grades of movement, effects of mobilisation, the irritability factor and differences between pain arising from inflammation and mechanical lesions. After a demonstration, the course participants had the opportunity to practice examination and some mobilisation techniques of the cervical spine. Methods of treating some common disorders of the cervical spine by means of mobilisation techniques were discussed.

The second session dealt with Neurological Techniques. Mr. C. Liggins and Mrs. H. Tarr gave the philosophy and explained how Rood methods are used to obtain either facilitation or inhibition using stretch, resistance, vibration, brushing, icing, positioning, joint compression, movement, etc. A practical demonstration of brushing, icing and vibration to hemiplegics in the flaccid stage was given. They also showed how a spastic hand could be released. After stressing the importance of ankle dorsiflexion in the hemiplegic, practical facilitation of this was shown. The importance of weight-bearing on the affected limb was explained, followed by a practical demonstration of inhibition of a strong flexor withdrawal reflex in the lower limb. A discussion on the use of ice in bladder retention and incontinence followed. Finally hamstring release was demonstrated.

Focussing on spasticity in hemiplegia and cerebral palsy, Mrs. E. Burnett and Mr. P. Gounden introduced the neurophysiology of the developmental approach to therapy. A discussion on the principles of physiotherapy in the management of spasticity using NDT was 'followed by a demonstration of the application of these principles.

In the third session Mr. C. Liggins discussed possible reasons for pain and its management. He suggested that traditional attitudes, difficulties of definition and poor organisation were some of the reasons that this was a relatively undeveloped clinical area. He discússed theories of pain such as specificity, pattern and gate control, as well as the role of enkephalin and the endorphins and the possible link between gate control theory and the endogenous opiates. The rest of the session concentrated on pain management. Physiotherapeutic modalities such as active and passive movement, massage, mobilisation, low frequency electrical stimulation, cold, "acupressure" and relaxation were mentioned and possible mechanisms explained.

Miss S. Blackwood, Mr. P. Gounden and Miss $\mathbf{H}$. Wilson presented aspects of paediatric, medical and surgical respiratory therapy, in the fourth session. A comparison was made between the mechanics of respiration in the adult and the infant, discussing the anatomical and physiological disadvantage of the infant respiratory system with the therapeutic modifications necessary. Therapeutic modalities relevant to the treatment of the child were included. A review of recent literature on aspects of physiotherapy in respiratory conditions lead to a discussion on the various methods of administration of inhaled bronchodilators; the efficacy of postural drainage, vibrations, percussion and shaking; the effect of localised lower lateral costal breathing in normal distribution of ventilation; the effectiveness of an exercise training programme improv- ing exercise tolerance whilst heart rate and lung function remain unaffected, and a method for teaching patients with chronic obstructive airway disease effective coughing. Charts to demonstrate general trends in patient condition were described. The long and short term effects of intermittent positive pressure breathing in the physiotherapy context were discussed. Slides were shown to illustrate trauma to the chest, surface markings and techniques of thoracotomy. The more common thoracic surgery was highlighted. The importance of physiotherapy in both preoperative and postoperative management, by physical means such as breathing exercises, coughing, support of the wound, limb and trunk exercises, as well as psychological pres paration even in small children were stressed. Within an hour of return to consciousness the patient should be stimulated to move the limbs, to breathe and to cough, augmented by vibrations and shaking, even when attached to a ventilator. Early ambulation should be encouraged consistent with the age and condition of the patient.

ln session five $\mathrm{Mr}$. C. A. Liggins gave the history of cold as a therapy and described its physiological effects. These were related to the various methods of therapeutic application of and the contraindications to cold. A practical demonstration of methods of conductive and evaporative cooling was followed by course participants practising these methods themselves.

In session six Mrs. J. Morton and Miss S. Eager dealt with sports injuries by commenting on the function of sport from the early Greeks to the modern Olympics. Aspects of the physiology of physical activity were highlighted, including maximum oxygen intake and energy utilisation during physical activity. The effect of training on glycogen utilisation, the effects of environment and stress, and fluid intake, were mentioned. Increasing participation in sport has led to an increase in the number of sport injuries. Whereas in a non-sporting person a joint strain can be immobilised, in a sportsman immobilisation should be avoided at all costs and if necessary, the patient should be taught a training routine which he can still do to maintain his level of fitness.

Acute diagnosis of injuries such as muscle and tendon tears and strains; haematoma and contusions; joint injuries with cartilage, capsule and ligamentous involvement; fractures and bony haematoma are of prime importance to assess whether the injury is to contractile or non-contractile tissue. Treatment starts with firstaid and then during the first 48 hours should include: rest - to take away strain from the area; ice — to reduce the amount of bleeding; compression - to reduce the bleeding and swelling; elevation - to reduce swelling and pain, and ultrasound - should be pulsed and at a low dose. After 48 hours heat and deep transverse frictions can be started, as well as stretching and graded exercises. The main aims of treatment are to regain mobility, increase endurance and strength, and to reeducate coordination.

The refresher course attracted a great deal of interest from physiotherapists from all parts of Natal and there were more applications than could be accommodated. All the speakers were Branch members and profits of the course will go to the PPK fund and towards financing future Branch academic activities. All who participated felt that the course was invaluable. 